\title{
Lidil
}

Revue de linguistique et de didactique des langues

\section{Le traitement de la finale du mot graphique par l'apprenti}

Un seuil cognitif entre apprentissage et expertise

Jean-Michel Sandon

\section{OpenEdition}

\section{Journals}

Édition électronique

URL : http://journals.openedition.org/lidil/923

DOI : $10.4000 /$ lidil. 923

ISSN : 1960-6052

Éditeur

UGA Éditions/Université Grenoble Alpes

Édition imprimée

Date de publication : 1 décembre 2004

Pagination : 169-182

ISBN : 2-914176-11-2

ISSN : 1146-6480

Référence électronique

Jean-Michel Sandon, « Le traitement de la finale du mot graphique par l'apprenti », Lidil [En ligne],

30 | 2004, mis en ligne le 29 janvier 2008, consulté le 30 avril 2019. URL : http://

journals.openedition.org/lidil/923; DOI : 10.4000/lidil.923

(c) Lidil 


\title{
LE TRAITEMENT DE LA FINALE \\ DU MOT GRAPHIQUE PAR L'APPRENTI
}

\author{
UN SEUIL COGNITIF ENTRE APPRENTISSAGE \\ ET EXPERTISE?
}

Jean-Michel SANDON *

Après des années d'étude de l'acquisition de l'écrit sous l'angle de la réception, les années quatre-vingt ont vu apparaitre des modèles de la production. Une dizaine d'années plus tard, les recherches se sont écartées d'une problématique fondée sur la seule correspondance sons-lettres. Désormais, la morphologie s'impose dans les recherches et il en va de même du traitement par les élèves de cette dimension spécifique de la modalité écrite en français. Cependant, les marques grammaticales retiennent toujours plus l'attention des psycholinguistes que les lettres à valeur lexicale. L'objet de cette étude est donc d'analyser quelques données relatives à la manière dont les 7-11 ans abordent certains aspects de la morphologie lexicale, d'en saisir les conséquences éventuelles sur la production et le contrôle en relecture des mots dans leurs textes.

Limité à cet aspect de la morphologie, cet article cherche d'abord à mettre en évidence les principaux traits caractéristiques du mot graphique français à l'intérieur duquel certaines lettres reçoivent une valeur activée par leur présence dans la famille lexicale (ex: enfant/enfantin). Il aborde ensuite la question de l'émergence d'une conscience de cette valeur et de son rôle dans l'évolution du comportement du scripteur en

* IUFM de Bourgogne. 
y distinguant des causes internes à la langue (ou endogènes) et d'autres relevant de l'enseignement (exogènes).

\section{Le mot graphique français : une évidence trompeuse}

Partant après bien d'autres de l'idée que l'écriture est plus une analyse de la langue que sa transcription (Olson, 1998; Cerquiglini, 1996), nous conduisons notre étude dans le cadre de cette unité complexe et suspecte aux yeux des linguistes qu'est le mot graphique (Pergnier, $1986: 16$ ).

Fondement de toute orthographe, le mot est un lieu de recomposition des unités de la langue. C'est la première des unités de sens dans un médium qui s'impose d'abord comme codage visuel de l'oral par les unités phonographiques. Celles-ci y sont souvent réexaminées («in»> «ain») par recours à des graphies ('a') que le sens du mot (plain/plaine $\leftrightarrow$ plan) justifie certes mais que les aléas des contextes socioculturels et historiques obscurcissent souvent par l'introduction de conventions (huître/ostréiculteur).

S'il s'impose au plan perceptif entre deux blancs graphiques et au plan cognitif par le ou les sens qu'il manifeste, sa structure interne ne se laisse pas percer aisément. Pour l'expert, c'est sa capacité interne d'évocation sémantique qui l'impose comme unité incontestable parce qu'efficace dans la communication écrite. Pour l'apprenti écrivant en revanche, produire une unité aussi complexe invite à la recherche de solutions de reconstructions qui, pour provisoires qu'elles soient, n'en dégagent pas moins des traits caractéristiques de notre écriture. Chercher à comprendre la part que prend la morphographie lexicale dans l'écriture du mot doit donc nous conduire à mettre en rapport le poids cognitif de telles unités pour les apprentis et la part qu'elles assument dans la dynamique interne au système graphique.

D'un point de vue structural, le mot graphique, unité composante de la syntaxe, reçoit en finale absolue des marques grammaticales spécifiques de la catégorie syntaxique qu'il y manifeste. Mais il est en même temps une unité composée (Gruaz, 1990:14) dont la hiérarchie interne peut être décrite ainsi : 
- éléments porteurs de sens :

- morphèmes dans le plan du contenu: un noyau, le radical (ex: «pièce») et des satellites: préfixes (ex: «en/em») et suffixes («ment») forment un mot construit («empiècement $»$ ),

- lettres dans le plan de l'expression: marques dérivatives directes (plat/plate) ou indirectes (souris/souriceau), finales (lit) ou internes (beau/belle), adjointes (pain) ou amalgamées à d'autres (air),

- éléments non porteurs de sens:

- éléments à fonction phonographique (ex: papa),

- éléments de liaison: pâtiss(i)er,

- lettres non-fonctionnelles: temp(s) - (h)uile.

Selon le point de vue - génétique -, qui est le nôtre, la morphologie lexicale est observée dans les commentaires métagraphiques (Jaffré et Ducard, 1996) des apprentis écrivants. Son émergence dans les justifications de formes produites ou corrigées est prise comme indice d'une logique segmentale qui ne s'appuie plus uniquement sur la transcription des sons, sur la mémoire du mot ou sur le raisonnement grammatical. L'importance numérique de sa présence par rapport à celle de ces autres appuis et l'indépendance de son existence par rapport à ceux-ci nous informent sur son accessibilité cognitive et sur son rôle effectif dans le fonctionnement du système.

\section{L'acquisition}

\section{Méthodologie}

L'analyse conduite ici s'appuie sur une étude plus large, à la fois longitudinale et transversale.

Le recueil de données se fait en classe à partir des écrits individuels de premier jet d'élèves de l'école élémentaire (de la $2^{\mathrm{e}}$ à la $5^{\mathrm{e}}$ année). Soixante seize élèves contrastés selon l'origine socio-professionnelle des parents et selon leurs performances en lecture et en production écrite (Lennox et Siegel, 1994) sont observés toutes les sept semaines durant une période de un à quatre ans. L'observation est conduite durant 
une tâche de production individuelle de texte choisie par le maître de la classe en fonction de son projet du moment. L'observation proprement dite est suivie d'un entretien légèrement différé. Observation et entretien sont pris en charge par le chercheur qui n'intervient pas sur les choix didactiques de l'enseignant. Au cours de l'entretien, l'élève commente ses choix orthographiques à la demande de l'observateur qui ne lui fournit en retour aucune sanction pragmatique, ni positive ni négative. Les faits retenus dépendent des observations faites durant la tâche d'écriture et d'un souci de conserver dans le corpus un équilibre de représentation de tous les principes de fonctionnement du système graphique français. La faible représentation d'un domaine est donc déjà en soit un indice.

\section{Résultats : approche quantitative}

Deux analyses sont conduites pour penser la place que la morphologie lexicale occupe dans le traitement conscient des faits orthographiques chez l'élève de l'école élémentaire.

Une première compare la quantité de traitements relevant de ce domaine linguistique opérés par l'apprenti à d'autres traitements dont on peut penser qu'ils sont plus souvent utilisés pour justifier ou corriger une forme de mot.

Le recours à la morphologie lexicale pouvant s'exercer sur n'importe quelle partie du mot écrit, la seconde analyse vérifie l'hypothèse que ce travail s'effectue surtout en finale, lorsque l'unité dont la présence pose question n'a pas de valeur phonographique (lourd).

Seul le corpus complet qui fournit 270 commentaires métagraphiques pour ce domaine permet de faire une comparaison en pourcentages qui ait quelque signification. Un corpus aussi réduit quand on le rapporte à quatre années de production écrite ne peut nous permettre de conduire une analyse développementale stricte. Nous nous limiterons donc à des éclairages ponctuels mettant en évidence des évolutions récurrentes dont nous montrerons le caractère significatif.

\section{Première analyse}

La place réduite qu'occupe chez le jeune enfant le recours au principe morphologique dans le domaine lexical est large- 
ment confirmée. La justification de son écriture par l'image du mot («lait, ça s'écrit comme ça») ou par un ou plusieurs éléments représentatifs de celui-ci («il y a souvent des 't'à la fin des mots») dépasse les $42 \%$ de l'ensemble des explications fournies. L'appel à la fonction phonographique d'un élément n'est déjà plus que de $15 \%$ («j'ai mis «ai» pour faire [E]») et l'appel à une fonction morphographique dans le lexique («il faut ' $t$ ' parce qu' on dit "laitier"») n'atteint pas les $3 \%$. Si ce résultat confirme que l'élève au sortir immédiat de l'apprentissage formel de la lecture (CE1) ne cherche déjà plus la solution à ses problèmes d'écriture dans la seule transcription de l'oral, il montre aussi que la forme de mot produite trouve le plus souvent sa justification ailleurs. Elle la trouve soit en elle-même (image du mot: lait), soit dans la fréquence d'emploi d'un graphème ou d'un combinat graphémique (probabilité graphique: ' $t$ ' plus probable qu'une autre lettre) plutôt que dans un réseau de mots liés par des marques graphiques avec ou sans correspondant phonique (lait-laitier; laiterie; voire lacté plus tard pour le «ai »).

Trois séries de faits peuvent expliquer cela:

- une première implique l'écrit lui-même: le principe morphogrammique lexical n'est pas inscrit au cour même du système graphique français. Il relève "plus de tendances que de lois, de microsystèmes que de systèmes» (Catach et al., 1980 : 247). En outre, de telles marques étant très souvent pluri-fonctionnelles, leur rôle tend à se fondre soit dans la transcription phonémique soit dans l'image du mot lui-même (tout en transcrivant un son, «ain» de «pain» renvoie à «panification» mais s'oppose aussi à «pin»);

- une seconde implique l'apprenti écrivant: avec ce fonctionnement qui les caractérise, les marques de la morphologie lexicale à l'écrit ne semblent pas très accessibles au plan cognitif. Leur productivité est faible et les liens qu'elles établissent en dehors des plus étroits d'entre eux (masculin féminin en particulier) ne correspondent pas au sens du développement du lexique chez l'enfant («main» est appris à l'écrit longtemps avant que «manuel» ne soit disponible dans le lexique actif) ;

- la troisième série est de l'ordre de la didactique de l'écrit: 
le lexique reste le parent pauvre de l'école élémentaire et les raisonnements analogiques (grand < grande), utilisés à l'oral dans les débuts de l'enseignement, sont encore insuffisamment développés sous une présentation écrite pour permettre à tous de trouver des angles d'observation du mot graphique complémentaires de l'entrée par les phonogrammes (Rilliard et Sandon, $1994: 53$ ).

\section{Seconde analyse}

Dans $73 \%$ des traitements relevant de la morphologie lexicale le contrôle s'effectue sur la lettre finale du mot avec parfois confusion entre marque grammaticale et marque relevant de ce domaine ( bois, il faut ' $s$ ' parce qu'il y en a beaucoup »). Mais, souvent, une marque grammaticale qui existe est reconnue comme telle. Elle est alors écartée du système explicatif qui conduit l'apprenti à justifier la fin d'un mot en la liant à un mot de la même famille.

Comme on pouvait s'y attendre - dans le système graphique français les liens les plus étroits sont installés entre formes du masculin et formes du féminin correspondantes -, c'est aussi là que les commentaires des élèves sont les plus nombreux et en général les plus pertinents.

\section{Résultats : approche qualitative}

La stabilité préservée du mot graphique: importance des sources endogènes du traitement de la morphologie lexicale à l'écrit.

Nous avons pu montrer ailleurs (Sandon, 2003) que, passé le temps formel de l'apprentissage de la lecture, l'association d'un phonogramme plausible et d'un phonème est une procédure élémentaire qui mobilise peu la conscience de l'élève. L'essentiel de son attention se concentre très tôt sur d'autres aspects et particulièrement sur des unités qui contribuent à conférer une légitimité graphique aux mots. Les conséquences en sont aussi importantes pour la suite de l'acquisition que celles qui suivent le passage au premier plan du principe phonographique chez les plus jeunes. Le contrôle du maintien du sens attaché au mot phonique par la simple transcription de celui-ci devient d'abord respect de l'image des 
mots connus de celui qui écrit. Momentanément, ce déplacement est créateur de formes qui font perdre à l'expression lexicale produite sa charge sémantique:

Jean Baptiste ; 8,04

Cest instrument avec les quels joue cest musicien sont le piano, le cor, la flute, le violon, le violon sel, la harpe

Je l'ai jamais vu écrit ce mot, je crois que ça s'écrit comme ça: il y a "violon», je le connais bien, et «sel», je le connais aussi.

Il suffit pourtant que ces mots constituent une chaine phonique identique au mot recherché pour que l'élève les considère légitimes.

L'apprenti cherche aussi à retrouver un élément existant en langue sans toutefois qu'on puisse déceler dans la relecture la conséquence d'une analyse ferme:

Florence ; 7,09

ca se cè denai dê cou de piê

Je l'ai barré; je croyais que le «de», il était pas attaché.

Quand on écrit des fois le "de», il n'est pas attaché aux autres mots.

Mais la forme phonique qui prend dans l'ontogenèse le statut de simple garant de l'écriture et la sémiographie qui émerge dans l'activité cognitive de l'élève sont déjà inscrites dans le fonctionnement même de l'orthographe française (Jaffré, 2001). C'est ce que retrouve l'élève, lorsque, confronté à la nécessité de spécifier de plus en plus les formes graphiques hors de tout étayage par l'adulte, il prend conscience que la phonographie ne peut seule assurer la reproduction de l'usage.

Jordane; 7,08

ma maman pous vai pas halai au travaille parcques dans note coure il avai du vergla

Je n'étais pas tout à fait sûr mais je savais qu'il fallait une lettre à la fin et je ne pouvais pas mettre un ' $x$ ' parce que $<$ poux $>$, c'est la petite bête alors j'ai écrit < pous >.

Anthony; 8,09

il lui tien les sel pour ne pas qu'il sent vol 
«s'envole», le «sent», je le connaissais, je l'ai déjà écrit, mais $<$ vol $>$, je ne savais pas lequel c'était, parce qu'il y a aussi le voleur, alors là, je n'ai pas mis de 'e'.

En se développant et en s'affinant, cette activité de réanalyse de la structure des mots conduit l'élève à focaliser son attention sur des marques sans fonction phonographique mais participant à la charge sémiotique du mot (Gruaz, 2003). Dans les cas cités - deux lecteurs et écrivants faibles -, la levée d'une ambigüité lexicale prime. Il s'agit de préserver le sens du mot transcrit. On a certes affaire à l'usage accepté d'une convention graphique mais on peut considérer, à partir d'autres études convergentes portant sur un autre principe du système (Sandon, 2002), qu'elle ne s'impose que parce qu'elle aide à la compréhension. Celui qui écrit conserve au mot son sens par un traitement de sa structure au-delà de la seule fonction phonographique de ses constituants. Les éléments ainsi ajoutés assument une fonction majeure: distinguer l'un de l'autre les mots phoniques qui appartiennent à la partie mobilisable du répertoire lexical de celui qui écrit.

Il reste alors à comprendre comment se produit un nouveau déplacement de l'attention vers des éléments qui participent aussi de la charge sémiotique du mot, mais au titre d'informations complémentaires, ayant un tout autre statut dans le système: les marques de la morphologie lexicale, rappels de l'existence d'une famille de mots.

C'est en effet ce déplacement du contrôle que semble effectuer Claire en cherchant à conserver un lien structurel in absentia:

Claire; 9,04

Je vais pas te dérangée plus longtemps

J'ai hésité pour le ' $\mathrm{g}$ ', et puis j'ai pensé à «long», et je me suis dit que c'était un mot de la même famille, alors qu'on écrivait ce mot et qu'on ajoutait un suffixe, le mot «temps».

Aucun souci de différencier ce mot d'un autre mot ici. Ne pas perdre le contact avec un mot de la même famille : c'est ce qui oriente le contrôle, au moins si l'on en juge par le commentaire. Mais ce traitement ne constitue sans doute pas encore un saut qualitatif fondamental. La structure du mot est en effet telle que la similitude entre éléments touche plus le 
mot entier «long» qu'une matrice graphique qui relierait par l'expression seule plusieurs mots ayant des liens de contenu.

En finale du mot graphique: la morphologie lexicale entre sources endogènes et sources exogènes.

Longtemps avant de pouvoir en défendre la légalité au plan grammatical comme au plan lexical, l'apprenti écrivant cherche donc à légitimer les formes des mots qu'il produit. Il focalise tôt son attention sur la finale des mots et la recherche qu'il y fait d'une stabilité justifiée grammaticalement contribue fortement à le faire changer de point de vue sur l'écrit. Mais ce contrôle qui se concentre sur cette zone du mot n'est pas sans conséquence sur la recherche d'éléments stables susceptibles de participer à la justification des choix graphiques que fait l'élève (Sandon, 2002). D'un mot à l'autre, indépendamment des marques grammaticales qui s'y ajoutent, des retours de lettres ou de combinats s'imposent, créant le sentiment que la zone de stabilité du mot graphique se trouve avant tout là, nouvelle légitimité pour les choix à faire.

Les premiers traitements de ce type sont d'ailleurs inconscients et comme irrépressibles. Qu'ils ne relèvent que de la pure analogie terme à terme comme chez Jordane:

Jordane; 9,08

Moi je pense que les animaux ses j(a)entils

Oh, c'est bizarre comme j'ai écrit la fin ici. J'ai dú confondre avec «outil».

ou qu'ils s'imposent malgré une autre connaissance prégnante, mémoire de mot comme chez Sébastien ou forme phonique comme chez Anne-Perrine :

Sébastien ; 7,02

sa mamant ariva.

J'ai écrit trop vite. Il y a beaucoup de mots qui s'écrivent avec «ant» à la fin. Mais «maman» je sais. Je savais déjà l'écrire à la maternelle.

Anne-Perrine ; 9,07

les (cheveaux) cheveux très courts

C'est bizarre parce que ce mot je le connais bien et je n'arrivais plus à écrire la fin. «eaux», c'est venu tout seul, mais après j'ai corrigé, parce que ça ne peut pas faire [ø]. 
Quand ces traitements deviennent conscients, c'est la fréquence perçue des rencontres dans le lexique utilisé qui oriente les choix. Des combinats graphiques s'imposent, à juste titre ou non, parce que, nonobstant leurs fonctions, l'apprenti peut les considérer comme des unités fréquentes dans le système.

Bertrand; 8,03

il et gentie

Je savais pas s'il y avait un 'e' ou pas; je ne suis jamais sûr de moi, alors j'écris souvent avec un ' $e$ ' à la fin après les 'é' et les ' $i$ ' parce qu'il y a souvent des 'e' à la fin des mots comme ça.

Jean Baptiste ; 8,01

Saiz années passerre

Là j'ai rajouté un ' $r$ ', mais je me suis trompé, c'est «ère». On voit ça toujours «mère; père; frère; gruyère " et puis je sais plus mais plein d'autres encore.

Puis, très tôt quand il s'agit de lier masculin et féminin, les explications fournies par les adultes se lient au contrôle par la légitimité graphique comme chez Jordane, ou s'installent indépendamment d'elle comme chez Bahar.

Jordane ; 9,1

il (s) était devenut le plus pauvre

Je le voyais mieux comme ça mais pourtant ça me gêne, parce que, au féminin, je ne vois pas bien un mot terminé en «ute».

Bahar; 10,05

c'est rigolot, c'est mignon

Au féminin, on peut dire «rigolote», alors il faut un ' $t$ ', et puis c'est souvent dans les mots, à la fin.

Le même traitement s'installe également très tôt et conduit à surgénéralisations dans le domaine du verbe. Extension analogique compréhensible chez Jean-Yves :

Jean-Yves ; 10,06

En rentrant à la maison il se pleind qu'il à mal au cou

Je l'ai écrit sans réfléchir. C'est le verbe «se plaindre», il se termine pas «dre», c'est comme "prendre».

ou véritable surgénéralisation chez Sébastien: 
Sébastien ; 8,03

On dit que je fais peur [...] Il fait Im 90

Ça je sais aussi que c'est «dit», que ça se finit par un ' $t$ ' parce que aussi on dit "dites-moi quelque chose». Et «je dis», c'est pareil. Et à la fin de «fait », je sais qu'il y a un ' $t$ ' parce qu'on dit «vous faites».

Le procédé semble alors servir de base pour justifier a posteriori d'autres finales verbales pourtant parfaitement régulières et parfois fréquentes en discours. Qu'il ne soit pas fait appel à la grammaire ou à la forme fréquente dans ces cas laisse penser que le procédé a plus une valeur symbolique dans l'échange avec l'adulte qu'il n'est réellement productif dans l'expression. Ce que tend à prouver un exemple comme celui-ci :

Marie; 8,03

elle prend sont temp et le chat l'atrapa

Si j'écrivais, «elle prendit» par exemple, on voit bien qu'il faudrait le 'd'.

Un pas de plus en revanche est franchi quand ce n'est plus l'appui sur la forme phonique du correspondant féminin ou une autre forme du verbe qui permet de trancher. Les commentaires gardent bien trace de l'enseignement reçu et de sa fragilité, mais les apprentis écrivants usent d'une convention qui, à l'écrit, dote le mot d'éléments d'information que ni le contexte sémantique ni le contexte syntaxique ne requièrent. A la légitimité graphique fondée sur la fréquence d'éléments placés à la frontière droite du mot à stabiliser se substitue un traitement qui sollicite le système dans ce qu'il a de plus virtuel. Si le recours à la mémoire est encore bien présent chez Sébastien:

Sébastien; 8,03

Je suis la souris

Je l'ai déjà vu plusieurs fois ce mot, alors je l'ai mémorisé. Et puis je sais très bien que ça se termine par un 's', parce qu'on dit «un petit souriceau». Ça ne change jamais même quand il y a « une souris».

Louise est déjà au-delà de ce recours direct. Sa recherche porte sur les mots absents qui peuvent justifier l'écriture du mot qui fait obstacle: 
Louise ; 7,03

les bras

J'ai fait pareil, parce que je savais pas vraiment la fin. J'hésitais entre ' $s$ ' et 'c' parce que je m'étais rappelé que aussi, on pouvait dire «brassière » ou un autre mot, je sais plus.

Sébastien et Mounir présentent des cas - intéressants quand ils se produisent chez les plus jeunes -, de déplacement du contrôle sur l'initiale et les consonnes doubles:

Sébastien ; 8,04

Il habite dans des cavernes

Je ne savais pas bien pour le début, mais au tableau, il y avait «habitat», alors $j$ 'ai mis un ' $h$ '.

Mounir; 10,05

les cheveux de Marie se décoiffèrent

J'ai hésité: 'f' ou 'ff', mais j'ai pensé à coiffeur.

Mais les cas de contrôle de l'écriture qui utilisent le plus nettement des matrices susceptibles d'assurer le lien entre éléments de la même famille alors que ni la position en frontière de mot ni une partie phonique commune ne sont en jeu sont les plus rares et sont aussi le fait des meilleurs lecteurs dans l'échantillon observé :

Claire ; 9,07

il (sé) sait que se cheval a le sang pur.

«sait» j'ai repensé, c'est le verbe «savoir» alors il faut que ça commence pareil et après il faut faire le [E] alors j'ai écrit «sai » et comme c'est «il », j'ai mis un ' $t$ '.

Claire; 9,07

J'ai oublier de te dire qu'(quaprès) qu'après la piscine nous irons à la fête foraine

Je sais qu'à «forain», on écrit «ain» alors pour faire «foraine», on garde le «ai » pour faire $[E]$ et on met un ' $e$ ' pour faire [n].

Contrairement à ce qui se passe lorsque le jeune élève commence à s'emparer de certains aspects de la morphologie verbale pour réexaminer ses propres modes de contrôle de l'écrit, et, ce faisant, réexaminer la représentation qu'il a des fonc- 
tions des marques orthographiques, son traitement de la morphologie lexicale à l'écrit semble plutôt prolonger des procédures existantes qu'il ne les transforme fondamentalement.

Déjà peu présentes parmi les modes de contrôle utilisés, ces procédures jouent durant toute la scolarité élémentaire un rôle complémentaire des procédures visuographiques qui contribuent à stabiliser les formes de mots les plus fréquentes. La finale des mots parait être, en dehors des marques grammaticales, une partie plus stable parce que récurrente dans de grandes zones du lexique. Que le contrôle lexical en vienne à se focaliser sur elle n'est donc pas un changement négligeable pour l'évolution du regard porté sur le fonctionnement de l'écrit. Il ouvre sur les aspects les plus virtuels d'un système (les éléments communs à une série lexicale) et contribue de ce fait à diversifier les modes d'analyse qui sont à la base de la stabilité indispensable aux mots écrits d'une langue. Mais la difficulté de l'apprenti à utiliser ces composantes du système quand l'oral ne peut en étayer simplement le recours semble confirmer, via l'observation de l'acquisition, le caractère peu dynamique de la morphographie lexicale dans l'écriture du français : elle impose en permanence un mode de calcul orthographique là où l'usager qui l'ignore peut respecter la norme à moindre effort grâce à un traitement holistique.

Il n'en reste pas moins que l'intérêt de l'apprenti écrivant pour cette forme de stabilisation des formes écrites pourrait inciter l'école élémentaire à porter une attention renouvelée à des outils analogiques mettant mieux en évidence les éléments virtuels du système comme elle tente déjà plus souvent de le faire pour les éléments à fonction morphosyntaxique. Il y va, là aussi, du développement des compétences du lecteurscripteur.

\section{Bibliographie}

Catach, N., Gruaz, C. et Duprez, D. (1980): L'orthographe française, traité théorique et pratique, Paris, Nathan/Université.

Cerquiglini, B. (1996): Le roman de l'orthographe. Au paradis des mots, avant la faute 1150-1694, Paris, Hatier. 
Gruaz, C. (1990): Du signe au sens, Presses Universitaires de Rouen, 158.

Gruaz, C. (2003): Tripolarité du langage, sémiotique du mot et sémiographie, in C. Gruaz (dir.), Quand le mot fait signe. Pour une sémiotique de l'écrit, Dyalang, 325, Presses universitaires de Rouen, 13-43.

JAFFRÉ, J.-P. (2001): Observation sur les effets potentiels de la dimension phonographique, Actes du Colloque Psychologie cognitive et apprentissage de la lecture, Tunis, nov. 2000.

JAFFRÉ, J.-P. et DUCARD, D. (1996) : Approches génétiques et productions graphiques, in J. David et M. Fayol (dir.), «Comment étudier l'acquisition de l'écriture ?», Etudes de linguistique appliquée, 101, 87-98.

LENNOX, C. et SIEGEL, L.S. (1994): The role of phonological and orthographic processes in learning to spell, in D.A. Gordon et N.C. Ellis, Handbook of Spelling: Theory, Process and Intervention, Chichester, John Wiley and Sons, 93-110.

OLson, D.R. (1998): L'Univers de l'écrit; comment la culture écrite donne forme à la pensée, Paris, Retz pour la traduction française.

PERGNIER, M. (1986): Le mot, Paris, PUF.

Rilliard, J. et SANDON J.-M. (1994): L'orthographe pendant la production de textes écrits, in A. Angoujard (dir.), Savoir orthographier, Paris, Hachette-éducation, 39-57.

Sandon, J.-Mí. (à paraitre): Dynamique du français écrit et dynamique de son apprentissage, Actes du colloque InterIUFM du Nord-Est, Apprendre et enseigner à l'école primaire.

SANDON, J.-M. (2003): Le mot et la lettre chez l'apprenti écrivant: du signe de reconnaissance au signe linguistique, in C. Gruaz (dir.), Quand le mot fait signe. Pour une sémiotique de l'écrit, Dyalang, 325, Presses universitaires de Rouen, 149-178. 